\title{
THE SPATIAL EXTENT OF (U)LIRGS IN THE MID-INFRARED. II. FEATURE EMISSION
}

\author{
T. Díaz-Santos ${ }^{1,2}$, V. Charmandaris ${ }^{1,3,4}$, L. Armus ${ }^{2}$, S. Stierwalt ${ }^{2}$, S. HaAn ${ }^{2}$, J. M. Mazzarella ${ }^{5}$, J. H. Howell ${ }^{2}$, \\ S. Veilleux ${ }^{6}$, E. J. Murphy ${ }^{7}$, A. O. Petric ${ }^{2}$, P. Appleton ${ }^{8}$, A. S. Evans ${ }^{9}, 10$, D. B. Sanders ${ }^{11}$, and J. A. Surace ${ }^{2}$ \\ ${ }^{1}$ Department of Physics, University of Crete, GR-71003 Heraklion, Greece; tanio@ physics.uoc.gr \\ ${ }^{2}$ Spitzer Science Center, California Institute of Technology, MS 220-6, Pasadena, CA 91125, USA \\ ${ }^{3}$ IESL/FORTH - Department of Physics and Institute of Theoretical \& Computational Physics, 71003 Heraklion, Greece \\ ${ }^{4}$ Chercheur Associé, Observatoire de Paris, F-75014 Paris, France \\ ${ }^{5}$ Infrared Processing \& Analysis Center, MS 100-22, California Institute of Technology, Pasadena, CA 91125, USA \\ ${ }^{6}$ Department of Physics, University of Oregon, Eugene, OR 97403, USA \\ ${ }^{7}$ Observatories of the Carnegie Institution for Science, Pasadena, CA 91101, USA \\ ${ }^{8}$ NASA Herschel Science Center, IPAC, MS 100-22, California Institute of Technology, Pasadena, CA 91125, USA \\ ${ }^{9}$ Department of Astronomy, University of Virginia, Charlottesville, VA 22904, USA \\ ${ }^{10}$ National Radio Astronomy Observatory, Charlottesville, VA 22903, USA \\ ${ }^{11}$ Institute for Astronomy, University of Hawaii, Honolulu, HI 96822, USA \\ Received 2011 April 19; accepted 2011 July 27; published 2011 October 12
}

\begin{abstract}
We present results from the second part of our analysis of the extended mid-infrared (MIR) emission of the GOALS sample based on 5-14 $\mu \mathrm{m}$ low-resolution spectra obtained with the Infrared Spectrograph on Spitzer. We calculate the fraction of extended emission (FEE) as a function of wavelength for all galaxies in the sample, $\mathrm{FEE}_{\lambda}$, defined as the fraction of the emission that originates outside of the unresolved central component of a source, and spatially separate the MIR spectrum of a galaxy into its nuclear and extended components. We find that the [Ne II] $12.81 \mu \mathrm{m}$ emission line is as compact as the hot dust MIR continuum, while the polycyclic aromatic hydrocarbon (PAH) emission is more extended. In addition, the 6.2 and $7.7 \mu \mathrm{m}$ PAH emission is more compact than that of the $11.3 \mu \mathrm{m} \mathrm{PAH}$, which is consistent with the formers being enhanced in a more ionized medium. The presence of an active galactic nucleus (AGN) or a powerful nuclear starburst increases the compactness and the luminosity surface density of the hot dust MIR continuum, but has a negligible effect on the spatial extent of the PAH emission on kpc-scales. Furthermore, it appears that both processes, AGN and/or nuclear starburst, are indistinguishable in terms of how they modify the integrated PAH-to-continuum ratio of the FEE in (ultra)luminous infrared galaxies ((U)LIRGs). Globally, the 5-14 $\mu \mathrm{m}$ spectra of the extended emission component are homogeneous for all galaxies in the GOALS sample. This suggests that, independently of the spatial distribution of the various MIR features, the physical properties of star formation occurring at distances farther than $1.5 \mathrm{kpc}$ from the nuclei of (U)LIRGs are very similar, resembling local star-forming galaxies with $L_{\mathrm{IR}}<10^{11} L_{\odot}$, as well as star-formation-dominated ULIRGs at $z \sim 2$. In contrast, the MIR spectra of the nuclear component of local ULIRGs and LIRGs are very diverse. These results imply that the observed variety of the integrated MIR properties of local (U)LIRGs arise, on average, only from the processes that are taking place in their cores.
\end{abstract}

Key words: galaxies: active - galaxies: evolution - galaxies: interactions - galaxies: starburst - infrared: galaxies

Online-only material: color figures, machine-readable table

\section{INTRODUCTION}

The mid-infrared (MIR) spectra of galaxies provide key information that allow us to characterize the global properties of their star formation, as well as to develop continuum and emission line diagnostics in order to infer whether they harbor an active galactic nucleus (AGN), with less confusion due to dust extinction compared to observations at optical wavelengths (Lutz et al. 1998b; Genzel et al. 1998; Charmandaris et al. 2004; Brandl et al. 2006; Smith et al. 2007; Armus et al. 2007; Veilleux et al. 2009; Wu et al. 2009; Sales et al. 2010; Petric et al. 2011). However, when studying the integrated emission of galaxies, the spatial distributions of the sources contributing to their observed IR spectral energy distributions, such as $\mathrm{H}$ II regions, photodissociation regions (PDRs), cold molecular clouds, and/or non-thermal emission sources, are averaged together. That is, all the intrinsic properties of the different phases of the interstellar medium (ISM) are mixed, despite the fact that they can be substantially different (Laurent et al. 2000; Smith et al. 2004). Therefore, even a rough decomposition between the nuclear and disk components of a galaxy can yield valuable information on where the IR luminosity originates, as well as details on the processes associated with the different emission components.

The access to this spatial information is even more vital in the study of luminous and ultraluminous infrared galaxies, (U)LIRGs, for which it is known that the bulk of their energy production is generated within their central regions and emitted in the IR on scales of a few kiloparsecs. This class of galaxies, although not very numerous in the nearby universe (Sanders \& Mirabel 1996), is responsible for the bulk of the obscured star formation at $z \geqslant 1$ (Le Floc'h et al. 2005; Pérez-González et al. 2005; Caputi et al. 2007; Magnelli et al. 2011; Murphy et al. 2011). Thus, the study of the spectra of local (U)LIRGs, where the angular resolution achieved by spaceborne observatories such as Spitzer is sufficient to spatially resolve their components, is essential for interpreting the internal mechanisms that govern their IR emission and for helping to understand the properties of high-redshift (U)LIRGs. 
Several MIR spectroscopic studies have already been carried out using ground-based telescopes which provide, for the closest and brightest sources, spatial resolution of a few tens of parsecs (Soifer et al. 2002, 2003; Díaz-Santos et al. 2010a). These works have demonstrated that the MIR continuum emission, ionization lines, and polycyclic aromatic hydrocarbon (PAH) features arise from different regions in (U)LIRGs, and that the sources of emission are arranged in more compact configurations than in galaxies with lower IR luminosities. This is consistent with the merger-induced nature of most local (U)LIRGs (Veilleux et al. 2002; Armus et al. 2009) and the funneling of large quantities of gas and dust mass toward their nuclei during the interaction. In addition, MIR spectral maps using the InfraRed Spectrograph (IRS) instrument on board Spitzer have been obtained for 15 local LIRGs revealing an increase of the electron density in their nuclei (Pereira-Santaella et al. 2010).

Despite the small number of galaxies studied in these works, some general conclusions about the properties of (U)LIRGs as a whole could be derived, yet these trends are confounded by systematics caused by intrinsic variations of processes that dominate at sub-kiloparsec scales. Studying these internal processes is therefore essential if we wish to completely understand the global trends. The Great Observatories All-Sky LIRG Survey (GOALS; Armus et al. 2009), which consists of 291 galaxies (202 systems), is the ideal local LIRG sample for this task because it is large enough to allow us to break it up into subsamples based, for example, on their interaction stage, or the AGN contribution to their MIR emission (Petric et al. 2011). Each subsample has enough galaxies in order to perform a robust statistical analysis and examine how the various MIR properties vary from one subsample to another and within a given subsample. Such an analysis was performed by Díaz-Santos et al. (2010b), hereafter Paper I, who analyzed the compactness of the MIR continuum emission at $13.2 \mu \mathrm{m}$ of (U)LIRGs in the GOALS sample and discussed its correlation to several physical properties of the systems such as their $L_{\mathrm{IR}}$, MIR AGN fraction, and far-infrared (FIR) colors.

The wealth of information available in the 5-14 $\mu \mathrm{m}$ spectral range allows us to further expand our analysis to several other key MIR features. In this second paper, we explore how the compactness of the emission due to $\mathrm{PAH}$ and atomic lines compares to that of the warm dust continuum and we investigate the origin of the observed differences. An analysis regarding the nuclear PAH emission ratios among the GOALS sample will be further addressed in a following study by S. Stierwalt et al. (2011, in preparation). The paper is structured as follows: in Section 2 we briefly remind the reader of the data sets used and the methodology of our analysis which was described in detail in Paper I. Our results are reported in Section 3, where we present average MIR spectra of the extended and nuclear emission of the three main types of sources we found in Paper I. We also explore the role of dust extinction as well as the presence of an AGN in the observed spatial profiles of the various MIR spectral features. Finally, in Section 4 we present our conclusions.

\section{OBSERVATIONS AND DATA ANALYSIS}

\subsection{The Sample}

The sample we analyze is the GOALS (Armus et al. 2009). GOALS comprises a complete, flux-limited sample of galaxies in the local universe drawn from the Revised Bright Galaxy Sample (Sanders et al. 2003) selected to be systems in the (U)LIRG luminosity classes. Armus et al. (2009) describe in detail how the sample was selected as well as its global characteristics. In addition, using a number of MIR diagnostics, Petric et al. (2011) estimate the AGN contribution to the MIR luminosity of the systems and, based on their apparent morphology, classify each galaxy into a stage of interaction, ranging from isolated systems to advanced mergers. In Howell et al. (2010) the relation between the UV and MIR emission of the galaxies in the sample is investigated, while Haan et al. (2011) present a thorough analysis of the nuclear structure of the galaxy sample using high spatial resolution NIR and optical images obtained with the Hubble Space Telescope. We refer the reader to these papers since we will rely on their findings for the interpretation of our results. Out of the 291 galaxies (202 systems) included in the GOALS sample, a total of 221 are used for this study, 200 LIRGs and 21 ULIRGs. All of them have low-resolution spectroscopic Spitzer/IRS observations in the 5-14 $\mu \mathrm{m}$ rest-frame wavelength range. The angular resolution of IRS $(\sim 3$.' 6 at $13.2 \mu \mathrm{m})$ allows us to separate regions of physical scales ranging from $0.22 \mathrm{kpc}$ at the distance of the closest LIRG $(\sim 12 \mathrm{Mpc})$, to $6.1 \mathrm{kpc}$ at $\sim 340 \mathrm{Mpc}$ where the farthest ULIRG of the sample is located. The spatial resolution at the median distance of our galaxy sample, $91 \mathrm{Mpc}$, is $1.7 \mathrm{kpc}$ (also at $13.2 \mu \mathrm{m}$ ). A table with the main characteristics of the galaxies such as their distance, $L_{\mathrm{IR}}$, or FIR colors can be found in Paper I. We also analyzed separately a compilation of ULIRGs from the works of Imanishi et al. (2007, 2010) and Imanishi (2009), but these sources cannot be included in our present study because they are too distant and, as a consequence, they are unresolved at all wavelengths observed with the IRS.

\subsection{Analysis}

Our analysis is based on the calculation of the fraction of extended emission (FEE) of galaxies as a function of wavelength, $\mathrm{FEE}_{\lambda}$, which is defined as the fraction of emission in a galaxy that does not arise from its spatially unresolved central component. Detailed information on the calculation of the $\mathrm{FEE}_{\lambda}$ functions can be found in Paper I. For practical purposes, we remind the reader that its formal definition is:

$$
\mathrm{FEE}_{\lambda}=\frac{\mathrm{EE}_{\lambda}}{E_{\lambda}(\text { total })},
$$

where $\mathrm{FEE}_{\lambda}, \mathrm{EE}_{\lambda}$, and $E_{\lambda}$ (total) are the fraction extended emission (ranging from zero to unity), the extended emission, and the total emission of a galaxy within the IRS slit at each wavelength, respectively. Essentially, the $\mathrm{FEE}_{\lambda}$ of a source can be considered as the complementary part of its compactness, which can be defined as $1-\mathrm{FEE}_{\lambda}$. We also define the core size of a galaxy as the FWHM of a Gaussian fitted to the spatial profile of its nuclear emission along the Spitzer/IRS slit at a given wavelength. We note that while the core size represents how extended the nuclear emission is, the FEE also accounts for low surface brightness emission that is more extended than the core of the source (beyond the wings of the central Gaussian). In Table 1 we provide the $\mathrm{FEE}_{\lambda}$ measurements at the wavelengths of the MIR spectral features considered in this work.

\section{RESULTS AND DISCUSSION}

In Paper I we grouped the GOALS galaxies into three types of $\mathrm{FEE}_{\lambda}$ functions: constant/featureless, $\mathrm{PAH}$ - and line-extended, and silicate-extended. We excluded 8 sources that appeared unresolved, thus having an $\mathrm{FEE}_{5-14 \mu \mathrm{m}} \simeq 0$, and 11 that could 
Table 1

FEEs of Various MIR Features

\begin{tabular}{|c|c|c|c|c|c|c|c|c|c|}
\hline $\begin{array}{l}\text { Galaxy } \\
\text { Name } \\
\text { (1) }\end{array}$ & $\begin{array}{c}\text { R.A. } \\
\text { (J2000) } \\
(2)\end{array}$ & $\begin{array}{c}\text { Decl. } \\
(\mathrm{J} 2000) \\
(3)\end{array}$ & $\begin{array}{c}\mathrm{FEE}_{6.2 \mu \mathrm{m}} \\
(\mathrm{PAH}) \\
(4)\end{array}$ & $\begin{array}{c}\mathrm{FEE}_{6.7 \mu \mathrm{m}} \\
\text { (Cont.) } \\
(5)\end{array}$ & $\begin{array}{c}\mathrm{FEE}_{7.7 \mu \mathrm{m}} \\
(\mathrm{PAH}) \\
(6)\end{array}$ & $\begin{array}{c}\mathrm{FEE}_{9.7 \mu \mathrm{m}} \\
(\mathrm{PAH}) \\
(7)\end{array}$ & $\begin{array}{c}\mathrm{FEE}_{11.3 \mu \mathrm{m}} \\
\text { (Si abs.) } \\
(8)\end{array}$ & $\begin{array}{c}\mathrm{FEE}_{12.8 \mu \mathrm{m}} \\
([\mathrm{Ne} \mathrm{II}]) \\
(9)\end{array}$ & $\begin{array}{l}\mathrm{FEE}_{\lambda} \\
\text { Type } \\
(10)\end{array}$ \\
\hline NGC 0023 & $00^{\mathrm{h}} 09^{\mathrm{m}} 53^{\mathrm{s}} .35$ & $+25^{\circ} 55^{\mathrm{m}} 27^{\mathrm{s}} .8$ & $0.66 \pm 0.02$ & $0.65 \pm 0.01$ & $0.68 \pm 0.02$ & $0.66 \pm 0.01$ & $0.67 \pm 0.03$ & $0.68 \pm 0.02$ & 1 \\
\hline MCG-02-01-051 & $00^{\mathrm{h}} 18^{\mathrm{m}} 50^{\mathrm{s}} .90$ & $-10^{\circ} 22^{\mathrm{m}} 36^{\mathrm{s}} .8$ & $0.37 \pm 0.04$ & $0.38 \pm 0.02$ & $0.36 \pm 0.05$ & $0.40 \pm 0.02$ & $0.29 \pm 0.06$ & $0.43 \pm 0.03$ & 1 \\
\hline ESO350-IG038 & $00^{\mathrm{h}} 36^{\mathrm{m}} 52^{\mathrm{s}} .49$ & $-33^{\circ} 33^{\mathrm{m}} 17^{\mathrm{s}} .3$ & $0.08 \pm 0.04$ & $0.12 \pm 0.02$ & $0.13 \pm 0.04$ & $0.17 \pm 0.04$ & $0.10 \pm 0.06$ & $0.17 \pm 0.04$ & 2 \\
\hline NGC0232 & $00^{\mathrm{h}} 42^{\mathrm{m}} 45^{\mathrm{s}} .84$ & $-23^{\circ} 33^{\mathrm{m}} 41^{\mathrm{s}} .0$ & $0.36 \pm 0.07$ & $0.40 \pm 0.01$ & $0.49 \pm 0.04$ & $0.41 \pm 0.01$ & $0.38 \pm 0.05$ & $0.47 \pm 0.04$ & 3 \\
\hline NGC0232 & $00^{\mathrm{h}} 42^{\mathrm{m}} 52^{\mathrm{s}} .82$ & $-23^{\circ} 32^{\mathrm{m}} 28^{\mathrm{s}} .0$ & $0.28 \pm 0.09$ & $0.35 \pm 0.02$ & $0.29 \pm 0.07$ & $0.35 \pm 0.02$ & $0.22 \pm 0.06$ & $0.37 \pm 0.04$ & 2 \\
\hline $\mathrm{MCG}+12-02-001$ & $00^{\mathrm{h}} 54^{\mathrm{m}} 03^{\mathrm{s}} .89$ & $+73^{\circ} 05^{\mathrm{m}} 06^{\mathrm{s}} .0$ & $0.47 \pm 0.02$ & $0.47 \pm 0.04$ & $0.32 \pm 0.04$ & $0.50 \pm 0.01$ & $0.44 \pm 0.05$ & $0.49 \pm 0.04$ & 2 \\
\hline NGC 0317B & $00^{\mathrm{h}} 57^{\mathrm{m}} 40^{\mathrm{s}} .42$ & $+43^{\circ} 47^{\mathrm{m}} 32^{\mathrm{s}} .6$ & $0.25 \pm 0.06$ & $0.24 \pm 0.01$ & $0.36 \pm 0.06$ & $0.29 \pm 0.07$ & $0.19 \pm 0.07$ & $0.33 \pm 0.04$ & 3 \\
\hline MCG-03-04-014 & $01^{\mathrm{h}} 10^{\mathrm{m}} 08^{\mathrm{s}} .93$ & $-16^{\circ} 51^{\mathrm{m}} 10^{\mathrm{s}} .1$ & $0.50 \pm 0.04$ & $0.53 \pm 0.01$ & $0.58 \pm 0.03$ & $0.54 \pm 0.01$ & $0.53 \pm 0.04$ & $0.57 \pm 0.03$ & 1 \\
\hline ESO244-G012 & $01^{\mathrm{h}} 18^{\mathrm{m}} 08^{\mathrm{s}} .23$ & $-44^{\circ} 28^{\mathrm{m}} 00^{\mathrm{s}} .5$ & $0.16 \pm 0.05$ & $0.18 \pm 0.02$ & $0.20 \pm 0.03$ & $0.20 \pm 0.01$ & $0.12 \pm 0.05$ & $0.20 \pm 0.04$ & 1 \\
\hline CGCG436-030 & $01^{\mathrm{h}} 20^{\mathrm{m}} 02^{\mathrm{s}} \cdot 64$ & $+14^{\circ} 21^{\mathrm{m}} 42^{\mathrm{s}} .1$ & $0.17 \pm 0.06$ & $0.26 \pm 0.03$ & $0.36 \pm 0.06$ & $0.24 \pm 0.05$ & $0.07 \pm 0.07$ & $0.35 \pm 0.05$ & 3 \\
\hline
\end{tabular}

Notes. (1) Galaxy name. Multiple systems are indicated with the same name but the right ascension and declination of the individual galaxies is provided. An asterisk next to a name indicates that multiple nuclei, not resolved by Spitzer/IRS, are detected using higher angular resolution HST NIR continuum imaging (Haan et al. 2011). (2) Right ascension (J2000). (3) Declination (J2000). (4)-(9) Fraction of extended emission (FEE) at: 6.2, 6.7, 7.7, 9.7, 11.3, and 12.8 $\mu$ m, respectively. (10) $\mathrm{FEE}_{\lambda}$ function type: $0, \mathrm{FEE}_{\lambda} \sim 0$; 1, constant/featureless; 2, PAH-dominated; 3, silicate-dominated; 4, undefined.

(This table is available in its entirety in a machine-readable form in the online journal. A portion is shown here for guidance regarding its form and content.)

not be classified in any of the three main $\mathrm{FEE}_{\lambda}$ types. However, for completeness, in the present paper we do take them into account. Figure 1 shows examples of the $\mathrm{FEE}_{\lambda}$ function types.

\subsection{The FEE of Continuum, PAHs, and Emission Lines}

In Figure 2 we examine how the FEE of several MIR spectral features, averaged at the wavelength where they peak $\pm 0.1 \mu \mathrm{m}$, vary as a function of the FEE of the continuum emission at $13.2 \mu \mathrm{m}$, which was studied in Paper I. From left to right and top to bottom we display: the $11.3 \mu \mathrm{m}$ PAH FEE as a function of the FEE at the peak of the $9.7 \mu \mathrm{m}$ silicate feature, and the $6.6 \mu \mathrm{m}$ continuum FEE, [Ne II] $12.81 \mu \mathrm{m}$ emission line $(+12.5 \mu \mathrm{m}$ PAH) FEE, $11.3 \mu \mathrm{m}$ PAH FEE, $7.7 \mu \mathrm{m}$ PAH FEE, and $6.2 \mu \mathrm{m}$ PAH FEE as a function of the $13.2 \mu \mathrm{m}$ continuum FEE.

Figure 2(a) shows, in a quantitative way, the visual classification of the $\mathrm{FEE}_{\lambda}$ functions of galaxies performed in Paper I, which we used for separating sources with characteristic FEEs $\lambda$ in three main types/categories (Figure 1). Within the uncertainties, (U)LIRGs showing a constant/featureless $\mathrm{FEE}_{\lambda}$ (blue diamonds) are located on the one-to-one relation (black dashed line). Sources presenting an excess of extended PAH emission with respect to that of the continuum (green stars) are above the line, while galaxies with an $\mathrm{FEE}_{\lambda}$ peaking at $9.7 \mu \mathrm{m}$ (red circles) are generally located below. We refer the reader to Paper I for more details about this classification.

The principal reason we plot the $6.6 \mu \mathrm{m}$ continuum in Figure 2(b) is to verify that the loss of angular resolution as a function of wavelength that affects the 5-14 $\mu \mathrm{m}$ short-low spectra is not biasing our measurements of the $\mathrm{FEE}_{\lambda}$, such that it decreases as wavelength increases. We can see that this is not the case, as the best fit to the $\mathrm{FEE}_{6.6 \mu \mathrm{m}}$ and $\mathrm{FEE}_{13.2 \mu \mathrm{m}}$ data, both representing continuum emission, is compatible, within the uncertainty (see the gray shadow), with the one-to-one relation. This implies that the decrease in angular resolution as the wavelength increases does not affect the trends significantly, at least in terms of the FEE measurements, when enough galaxies are considered.

Figure 2(c) shows the FEE averaged at the location of the $\left[\mathrm{Ne}\right.$ II]12.81 $\mu \mathrm{m}$ emission line as a function of $\mathrm{FEE}_{13.2 \mu \mathrm{m}}$. Both quantities are well correlated, which is in agreement with previ- ous studies of smaller samples of local LIRGs (Pereira-Santaella et al. 2010), and implies that the physical processes (AGN and/ or star formation) that are responsible for the [Ne II] $12.81 \mu \mathrm{m}$ line and the MIR continuum emission are equally concentrated and that they are likely the same. On the other hand, the FEE of the $11.3 \mu \mathrm{m} \mathrm{PAH}$ is larger than that of the continuum or the $[\mathrm{Ne}$ II] $12.81 \mu \mathrm{m}$ emission in many galaxies (Figure 2(d)). Moreover, this is independent of the $\mathrm{FEE}_{\lambda}$ function type and does not only occur for silicate-dominated $\mathrm{FEE}_{\lambda}$ type galaxies (red circles; see related caveat in Section 3.2, where we discuss the effect of the extinction on the spatial distribution of the MIR emission of spectral features located within the wavelength range of the $9.7 \mu \mathrm{m}$ silicate absorption band). This result is in agreement with works based on high spatial resolution $N$-band $(8-13 \mu \mathrm{m})$ spectroscopy of local LIRGs where it has been found that the spatial distribution of the $[\mathrm{Ne}$ II $] 12.81 \mu \mathrm{m}$ emission and the MIR continuum in star-forming regions follows that of the Pa $\alpha$ emission (Díaz-Santos et al. 2010a), which is in turn associated with young, compact HII regions (e.g., Alonso-Herrero et al. 2002). Meanwhile, the $11.3 \mu \mathrm{m} \mathrm{PAH}$ emission can be powered not only by $\mathrm{H}$ II regions but also by rather "evolved" ( $\geq 8-10 \mathrm{Myr}$ ) stars, which emit fewer ionizing photons. In other words, the $11.3 \mu \mathrm{m} \mathrm{PAH} /[\mathrm{Ne}$ II $] 12.81 \mu \mathrm{m}$ flux ratio depends on the age of the ionizing stellar populations (Díaz-Santos et al. 2010a). This diffuse PAH emission would be located in between the circumnuclear young starforming regions and also farther from the nucleus of (U)LIRGs (Díaz-Santos et al. 2008; Pereira-Santaella et al. 2010; Bendo et al. 2010). One might think that, because of the limited spectral resolution of the data, the [Ne II] $12.81 \mu \mathrm{m}$ line could be contaminated by the emission of the $12.5 \mu \mathrm{m}$ PAH feature. However, this is not the case because if the $12.5 \mu \mathrm{m}$ PAH were as extended as the $11.3 \mu \mathrm{m}$ PAH, or even as much as the $7.7 \mu \mathrm{m}$ or $6.2 \mu \mathrm{m}$ PAHs (see below), and the $12.5 \mu \mathrm{m}$ PAH was dominating the emission at $12.8 \mu \mathrm{m}$ then, for a given value of the $\mathrm{FEE}_{13.2 \mu \mathrm{m}}$, the $\mathrm{FEE}_{12.8 \mu \mathrm{m}}$ should appear much more extended and/or with a larger scatter than what is observed in Figure 2(c). Instead, one can identify a tight correlation between both FEEs at $12.8 \mu \mathrm{m}$ and $13.2 \mu \mathrm{m}$.

Figures 2(e) and (f) show that the $7.7 \mu \mathrm{m}$ and $6.2 \mu \mathrm{m} \mathrm{PAH}$ emissions are as compact as, or just slightly more extended 


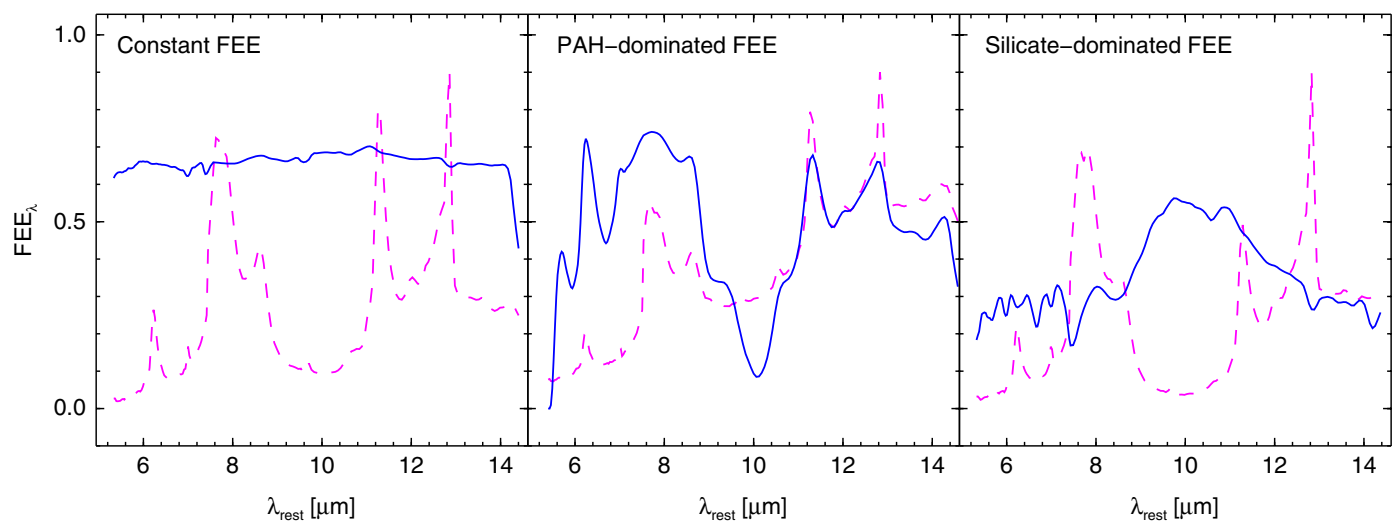

Figure 1. $\mathrm{FEE}_{\lambda}$ functions, smoothed with a 4 pixel box to reduce noise, are plotted in blue for three galaxies that serve as examples of the main types identified in the sample. The Spitzer/IRS 5-14 $\mu \mathrm{m}$ spectrum of each galaxy, scaled to arbitrary units, is also plotted as a pink dashed line for reference. Left panel: NGC 3110 with constant/featureless $\mathrm{FEE}_{\lambda}$. Middle panel: NGC 1365 displaying a PAH- and line-extended emission. Right panel: MCG+08-11-002 with silicate-extended emission. (A color version of this figure is available in the online journal.)

than, that of the MIR continuum and hence the $[\mathrm{Ne}$ II $] 12.81 \mu \mathrm{m}$ emission, but less than the $11.3 \mu \mathrm{m}$ PAH. This is in agreement with the notion that the $7.7 \mu \mathrm{m}$ and $6.2 \mu \mathrm{m}$ emission bands are enhanced for ionized PAHs, while the $11.3 \mu \mathrm{m}$ PAH emission is similar for neutral and ionized molecules (e.g., Allamandola et al. 1999; Galliano et al. 2008). Since ionized PAH carriers require harder radiation fields than neutral PAHs in order to be excited (Draine \& Li 2001), they will be more tightly associated with younger massive star-forming regions which, in turn, are also strong [Ne II] $12.81 \mu \mathrm{m}$ emitters. Given the nature of LIRGs and ULIRGs, many of which harbor strong nuclear starbursts, this would result in having both the $7.7 \mu \mathrm{m}$ and the $6.2 \mu \mathrm{m}$ PAHs slightly more extended than the $[\mathrm{Ne}$ II] $12.81 \mu \mathrm{m}$ line but less extended than the $11.3 \mu \mathrm{m}$ PAH.

In Paper I we also examined the variations of the FEE of the continuum at $13.2 \mu \mathrm{m}$ as a function of the $L_{\mathrm{IR}}$. Here we also explore how the FEE of PAHs in (U)LIRGs depends on the $L_{\mathrm{IR}}$ and compare them with their corresponding $\mathrm{FEE}_{13.2 \mu \mathrm{m}}$. Figure 3 shows that, despite the large dispersion, the median of the $\mathrm{FEE}_{7.7 \mu \mathrm{m}} / \mathrm{FEE}_{13.2 \mu \mathrm{m}}$ ratio (black solid line) slightly increases with $L_{\mathrm{IR}}$. The more IR luminous a galaxy is, the more extended its $11.3 \mu \mathrm{m}$ PAH emission is compared with the MIR continuum. This is in agreement with our earlier result in Paper I, where we found that the compactness of the MIR continuum increases for galaxies with $L_{\mathrm{IR}} \gtrsim 10^{11.8} L_{\odot}$. However, the $6.2 \mu \mathrm{m}, 7.7 \mu \mathrm{m}$, and $11.3 \mu \mathrm{m}$ PAHs do not show such a steep decrease of their FEEs around these IR luminosities. Therefore, it is mainly the MIR continuum that becomes more compact as the $L_{\mathrm{IR}}$ of galaxies increases, causing the median PAH-to-continuum FEE ratio to increase as well. Likewise, the most luminous galaxies are also those having the warmest $\log \left(f_{60 \mu \mathrm{m}} / f_{100 \mu \mathrm{m}}\right)$ FIR ratios. This is expected for ULIRGs, which are known to host nuclear starbursts and AGNs and show on average warm colors (Sanders \& Mirabel 1996), but these same properties are also displayed by several sources with lower IR luminosities $\left(10^{11} L_{\odot} \lesssim L_{\mathrm{IR}} \lesssim 10^{12} L_{\odot}\right)$. Indeed, independently of their $L_{\mathrm{IR}}$, the warmest galaxies, those with $\log \left(f_{60 \mu \mathrm{m}} / f_{100 \mu \mathrm{m}}\right)>-0.2$ (identified by red circles in Figure 3), are those driving the trend and accounting for the dispersion seen in the data. As we will discuss later, this is in agreement with their compact nature and the general scenario we will propose in Section 3.4.

\subsection{The Role of Dust Extinction}

We also investigated the specific distribution of (U)LIRGs of different $\mathrm{FEE}_{\lambda}$ types in Figures 2(a)-(e). As we mentioned above, Figure 2(c) shows that the $\mathrm{FEE}_{11.3 \mu \mathrm{m}}$ is larger than the $\mathrm{FEE}_{13.2 \mu \mathrm{m}}$ in many (U)LIRGs. However, for some of them this may be an extinction effect.

Even in the MIR, dust obscuration must be taken into account in (U)LIRGs since their nuclear extinction is much larger than that of their extended regions (e.g., García-Marín et al. 2009). Galaxies with a silicate-dominated $\mathrm{FEE}_{\lambda}$ type are marked as red circles in Figure 2. We observe that for these sources the $11.3 \mu \mathrm{m}$ PAH feature, which is located within the $9.7 \mu \mathrm{m}$ silicate absorption feature, appears more extended (i.e., have large $\mathrm{FEE}_{11.3 \mu \mathrm{m}}$ values). This is not because there is more PAH emission in the disks of these galaxies but instead because the emission is suppressed in their nuclei due to the extinction, as we already suggested in Paper I. Therefore, the differential extinction between the unresolved and extended components plays an important role in the "apparent" spatial distribution of the emission of the different MIR features in these galaxies. Sources with extreme differences between the extinction in their nuclei and extended regions (large $\Delta S_{\mathrm{Si}} 9.7 \mu \mathrm{m}$, see Section 3.5) will show an artificial excess in their $\mathrm{FEE}_{11.3 \mu \mathrm{m}} / \mathrm{FEE}_{13.2 \mu \mathrm{m}}$ ratio (see implications in next sections). Indeed, this also applies in general to all features within the $\sim 8-12.5 \mu \mathrm{m}$ wavelength range affected by the $9.7 \mu \mathrm{m}$ silicate feature, such as the $8.6 \mu \mathrm{m}$ PAH and the [S IV] $10.51 \mu \mathrm{m}$ line. As a consequence, in order to interpret correctly the FEE ratios of those galaxies with large $\Delta S_{\mathrm{Si} 9.7 \mu \mathrm{m}}$, a careful decomposition of their spectra in different dust components and feature emissions must be done to properly account for extinction effects (S. Stierwalt et al. 2011 , in preparation).

Nevertheless, not all (U)LIRGs have highly obscured nuclei at $9.7 \mu \mathrm{m}$. There are several galaxies in our sample, those with a $\mathrm{PAH}$-dominated $\mathrm{FEE}_{\lambda}$ type, that show high PAH-to-continuum FEE ratios but, unlike galaxies with silicate-dominated $\mathrm{FEE}_{\lambda}$ types, they do not display large differences between the extinction of their unresolved and extended components.

\subsection{The Influence of an $A G N$}

In Paper I we studied how the FEE of the continuum at $13.2 \mu \mathrm{m}$ varies with the MIR AGN fraction of galaxies and showed that the MIR continuum becomes more compact for AGN-dominated sources. In order to examine whether the presence of an AGN also influences the distribution of the PAH emission in (U)LIRGs, we plot in Figure 4 the histogram of the ratio of the $7.7 \mu \mathrm{m}$ PAH FEE to the $13.2 \mu \mathrm{m}$ 

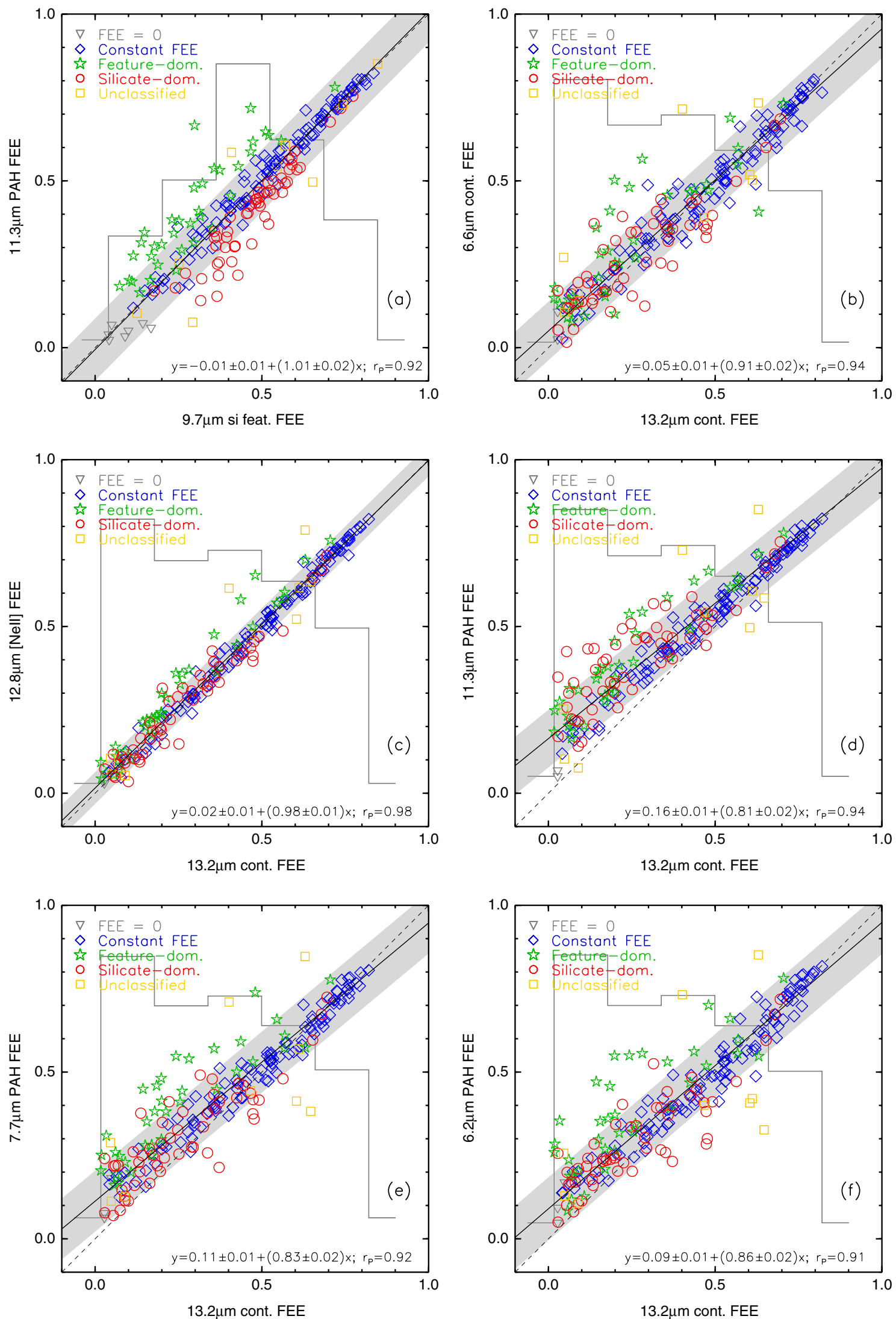

Figure 2. From left to right and top to bottom: (a) $\mathrm{FEE}_{11.3 \mu \mathrm{m}}\left(11.3 \mu \mathrm{m}\right.$ PAH) vs. FEE $9.7 \mu \mathrm{m}$ (peak of the silicate absorption feature). Then, $\mathrm{FEE}_{13.2 \mu \mathrm{m}}$ (continuum) vs.: (b) $\mathrm{FEE}_{6.6 \mu \mathrm{m}}$ (continuum), (c) $\mathrm{FEE}_{12.8 \mu \mathrm{m}}$ ([Ne II]12.81 $\mu \mathrm{m}$ line), (d) $\mathrm{FEE}_{11.3 \mu \mathrm{m}}$, (e) $\mathrm{FEE}_{7.7 \mu \mathrm{m}}(7.7 \mu \mathrm{m}$ PAH), and (f) FEE $6.2 \mu \mathrm{m}$ (6.2 $\mu \mathrm{m}$ PAH). The different symbols and colors represent the $\mathrm{FEE}_{\lambda}$ galaxy types (unresolved: dark gray inverted triangles; constant/featureless: blue diamonds; PAH-dominated: green stars; silicate-dominated: red circles; and unclassified: yellow squares). The black dashed line shows the one-to-one relation, while the black solid line is the linear fit to the data, whose parameters can be found in the bottom right of the plots. The shaded region is the $1 \sigma$ uncertainty of the fit, while the light gray underlying line is the arbitrarily scaled histogram of the data distributed in equal bins of $\mathrm{FEE}_{9.7 \mu \mathrm{m}}$ or $\mathrm{FEE}_{13.2 \mu \mathrm{m}}$.

(A color version of this figure is available in the online journal.) 


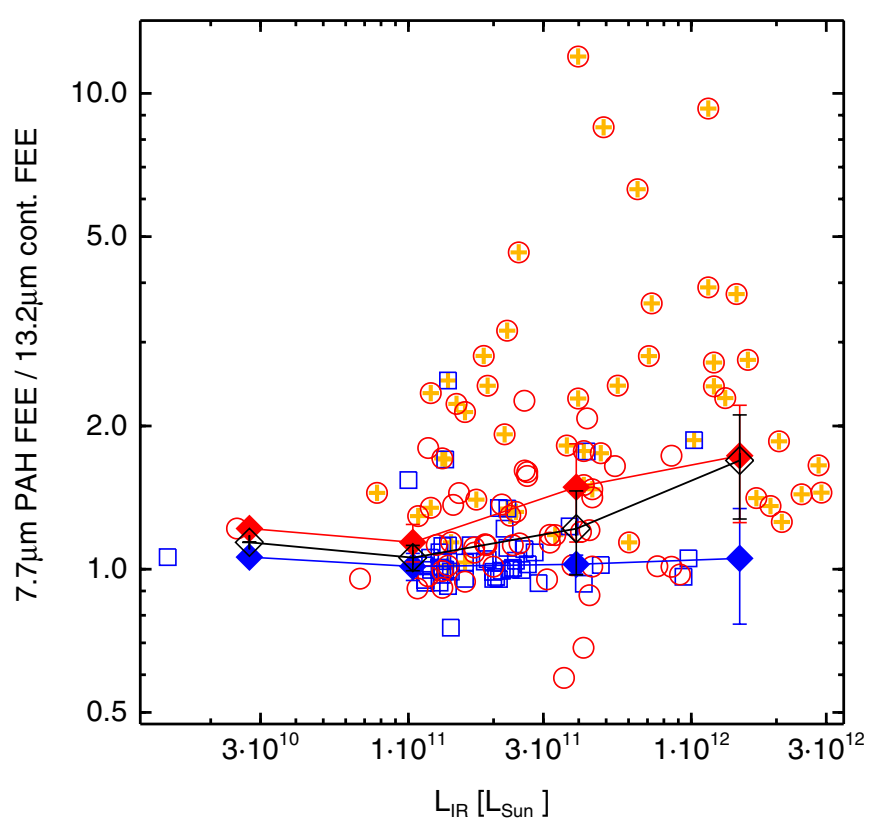

Figure 3. $\mathrm{FEE}_{7.7 \mu \mathrm{m}} / \mathrm{FEE}_{13.2 \mu \mathrm{m}}$ ratio as a function of the $L_{\mathrm{IR}}$ of our (U)LIRG sample. The symbols and colors depend on the IRAS $\log \left(f_{60 \mu \mathrm{m}} / f_{100 \mu \mathrm{m}}\right)$ FIR ratio of the galaxies. The blue squares and red circles represent cold and warm sources with $\log \left(f_{60 \mu \mathrm{m}} / f_{100 \mu \mathrm{m}}\right)$ ratios lower and larger than -0.2 , respectively. The blue and red filled diamonds show the median values of the $\mathrm{FEE}_{7.7 \mu \mathrm{m}} / \mathrm{FEE}_{13.2 \mu \mathrm{m}}$ ratio for the two groups of sources in equally distributed IR luminosity bins. The black diamonds represent the same ratio but considering all sources. Galaxies whose core sizes are unresolved at $13.2 \mu \mathrm{m}$ are marked with yellow crosses.

(A color version of this figure is available in the online journal.)

continuum FEE, grouping the galaxies as a function of their MIR AGN fraction. Galaxies in which an AGN dominates the MIR emission display large PAH-to-continuum FEE ratios. The median $7.7 \mu \mathrm{m}$ PAH-to-continuum FEE ratio for MIR star-formation-dominated galaxies, that is, sources for which Petric et al. (2011) find that the AGN contribution to the MIR emission is less than $50 \%$, is $1.08 \pm 0.10$, while for AGN-dominated sources, it is $1.86 \pm 0.41$. The corresponding median ratios for the $6.2 \mu \mathrm{m}$ PAH-to-continuum FEE ratio are $1.04 \pm 0.11$ and $1.74 \pm 0.51$, respectively. We note that a much larger difference is seen in the $\mathrm{FEE}_{11.3 \mu \mathrm{m}} / \mathrm{FEE}_{13.2 \mu \mathrm{m}}$ ratio. However, as discussed in Section 3.2, the $\mathrm{FEE}_{11.3 \mu \mathrm{m}}$ is affected by the $9.7 \mu \mathrm{m}$ silicate absorption feature and, at the same time, AGN-dominated galaxies tend to show large differences between the extinction of their nuclei and extended regions, i.e., large $\Delta S_{\mathrm{Si} 9.7 \mu \mathrm{m}}$ values. Both effects combined may artificially increase the $11.3 \mu \mathrm{m}$ PAH-to-continuum FEE ratio leading to a possible misinterpretation of the observed correlation. The 7.7 and $6.2 \mu \mathrm{m}$ PAH, on the other hand, do not suffer from severe obscuration and therefore the trend and ratios seen in Figure 4 are more reliable.

Although the difference between the median PAH-tocontinuum FEE ratios of MIR AGN- and star-formationdominated galaxies is at the $\sim 2 \sigma$ level, a Kolmogorov-Smirnov test performed between the two samples indicates clearly ( $p \sim$ $10^{-6}$ ) that the distributions are not drawn from same parent population. This, combined with the fact that the $\mathrm{FEE}_{13.2 \mu \mathrm{m}} \mathrm{de}-$ creases as the MIR AGN fraction increases (see Paper I) suggest that as the MIR emission of a (U)LIRG begins to be dominated by an AGN, the hot dust continuum becomes more compact while the PAH-emitting regions do not. That is, the presence of

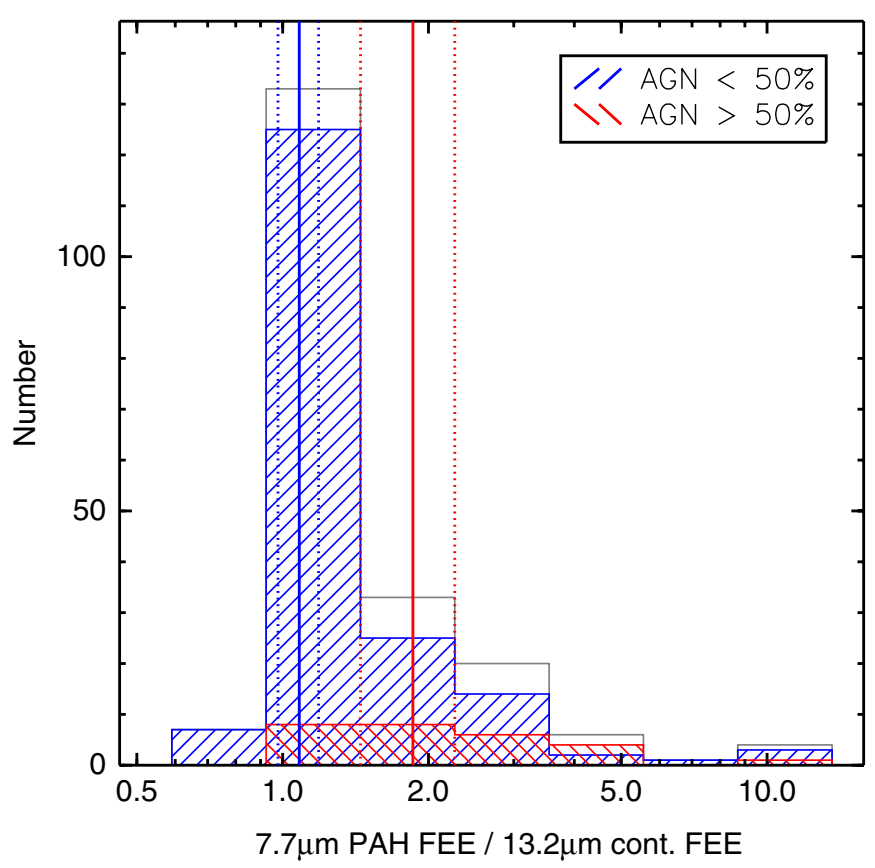

Figure 4. Histogram of the $\mathrm{FEE}_{7.7 \mu \mathrm{m}} / \mathrm{FEE}_{13.2 \mu \mathrm{m}}$ ratio of our galaxies grouped as a function of the AGN contribution to their MIR continuum. A total of 205 (U)LIRGs for which we have measurements of their $\mathrm{FEE}_{7.7} \mu \mathrm{m} / \mathrm{FEE}_{13.2} \mu \mathrm{m}$ ratios and AGN fractions from Petric et al. (2011) were used. The solid lines represent the median value of each distribution, while the dotted lines show the $\pm 1 \sigma$ uncertainty with respect to the medians. A Kolmogorov-Smirnov test indicates clearly $\left(p \sim 10^{-6}\right)$ that the distributions are not drawn from same parent population.

(A color version of this figure is available in the online journal.)

an AGN modifies the spatial extent of the hot dust continuum but has a negligible effect on the overall, kpc-scale distribution of the PAH emission.

Indeed, this is consistent with the way in which the AGN fraction is calculated in Petric et al. (2011). In this work the contribution of an AGN to the MIR emission of a (U)LIRG is estimated through the so-called Laurent diagram (Laurent et al. 2000; Armus et al. 2007) which, for the GOALS sample, relies mainly on the ratio between the $6.2 \mu \mathrm{m}$ PAH emission and the $5.5 \mu \mathrm{m}$ continuum (see Figure 3 in Petric et al. 2011). Therefore, as the compactness of the hot dust continuum increases, so does the surface brightness of the galaxy and, as a consequence, this pseudo-equivalent width (EW) of the $6.2 \mu \mathrm{m}$ PAH decreases, which implies that the AGN contribution to the MIR is larger and dilutes the PAH emission.

We have also investigated the influence of an AGN depending on the $\mathrm{FEE}_{\lambda}$ type of the host. For example, there are no galaxies with constant/featureless FEEs $_{\lambda}$ having an MIR AGN fraction above 0.5 . This is an important result since, as we have explained, the galaxies were classified into the different $\mathrm{FEE}_{\lambda}$ types independently of their MIR spectral characteristics. Thus, it appears that when an AGN dominates the MIR emission it also leaves a "footprint" in the $\mathrm{FEE}_{\lambda}$ function of a galaxy in the sense that the MIR emission of (U)LIRGs with a constant $\mathrm{FEE}_{\lambda}$ will never be dominated by an AGN or, in general, appear compact.

We would like to stress here that not only do AGN-dominated galaxies show large PAH-to-continuum FEE ratios but nearly $30 \%$ of (U)LIRGs whose MIR emission is not dominated by an AGN (AGN fraction < 0.5) display large $\mathrm{FEE}_{7.7 \mu \mathrm{m}} / \mathrm{FEE}_{13.2 \mu \mathrm{m}}$ ratios. The nature of these sources will be addressed in the next section. 


\subsection{PAH-to-continuum FEE Ratios and FIR Emission}

In this section we examine whether the PAH-to-continuum FEE ratios are related with the FIR colors of galaxies, that is, whether the differences in the compactness of the PAH and MIR continuum emission in (U)LIRGs are linked to their global dust temperatures traced by the FIR emission. We have already discussed that PAH emission probes timescales of star formation larger than the MIR continuum emission, which is tightly associated with young, ionizing stellar populations (e.g., Calzetti et al. 2007; Díaz-Santos et al. 2010a). Likewise, results based on IRAS as well as on recent high spatial resolution Herschel observations have clearly demonstrated that the $100-500 \mu \mathrm{m}$ emission of galaxies arises mainly from a diffuse cold dust component that extends smoothly along their disks and is heated by old stars, rather than being concentrated in knots of current star formation which are better traced by dust continuum emission at $<100 \mu \mathrm{m}$ (Sauvage \& Thuan 1992; Walterbos \& Greenawalt 1996; Bendo et al. 2010; Kramer et al. 2010). Moreover, in a recent Herschel study of local spiral galaxies it has been found that their cores show enhanced, warmer $70 / 160 \mu \mathrm{m}$ colors than their disks (Sauvage et al. 2010). Therefore, since in Paper I we showed that the FEE $13.2 \mu \mathrm{m}$ decreases when (U)LIRGs become warmer, i.e., when their IRAS $\log \left(f_{60 \mu \mathrm{m}} / f_{100 \mu \mathrm{m}}\right)$ FIR color increases, we would also expect to find a trend between the PAHto-continuum FEE ratios and the FIR color of galaxies.

Figure 5 shows that this is actually the case. Although the trend for the $6.2 \mu \mathrm{m}$ PAH-to-continuum FEE ratio is less significant, it is clear that the $7.7 \mu \mathrm{m}$ PAH-to-continuum FEE ratio and its dispersion increase as galaxies become warmer. Moreover, practically all (90\%) MIR star-formation-dominated sources with large PAH-to-continuum FEE ratios (sources above the dashed lines, which are defined as the medians $+2 \sigma$ of the distributions shown in Figure 4$)$ have $\log \left(f_{60 \mu \mathrm{m}} / f_{100 \mu \mathrm{m}}\right)$ $\gtrsim-0.2$. A plausible explanation for this is the following: as the nuclear starburst activity in a (U)LIRG increasingly dominates its integrated IR emission, the dust in the central regions would reach higher temperatures. This is because more and more of the atoms and molecules of the nuclear gas would be ionized and dissociated by the high-energy photons of the stars formed in the $\mathrm{H}$ II regions of their proximity, causing the ratio between the volume of the spherical shells of the PDRs, from where most of the PAH emission originate, and the volume of the dust-emitting region to decrease. That is, a larger fraction of the ionizing photons would be absorbed by dust close to the stars increasing its temperature, and would not reach the PDRs. As a result, the MIR continuum emission due to this hot dust will be more compact (having a higher luminosity surface density; see also Rujopakarn et al. 2011), and the global galaxy colors will become warmer.

We could then consider that the "normal" behavior of a galaxy is to show the same spatial extent of the PAH and MIR continuum emission probably as a consequence of a sufficient mixing of its gas and stars. It is only when a strong, compact emitter starts to dominate the nuclear MIR continuum that we see a deviation (i.e., PAH-to-continuum FEE ratios above the dashed lines in Figures 4 and 5). This is the case for $80 \%$ of AGN-dominated GOALS galaxies and $60 \%$ of warm sources with $\log \left(f_{60 \mu \mathrm{m}} / f_{100 \mu \mathrm{m}}\right)>-0.2$, excluding AGNdominated galaxies. In these systems, the MIR continuum is more compact than the PAH emission, which is still as extended as in the "normal" population since it arises mostly from a diffuse component (the disk of the galaxies). Therefore, a more appropriate interpretation would not be that there is an excess of
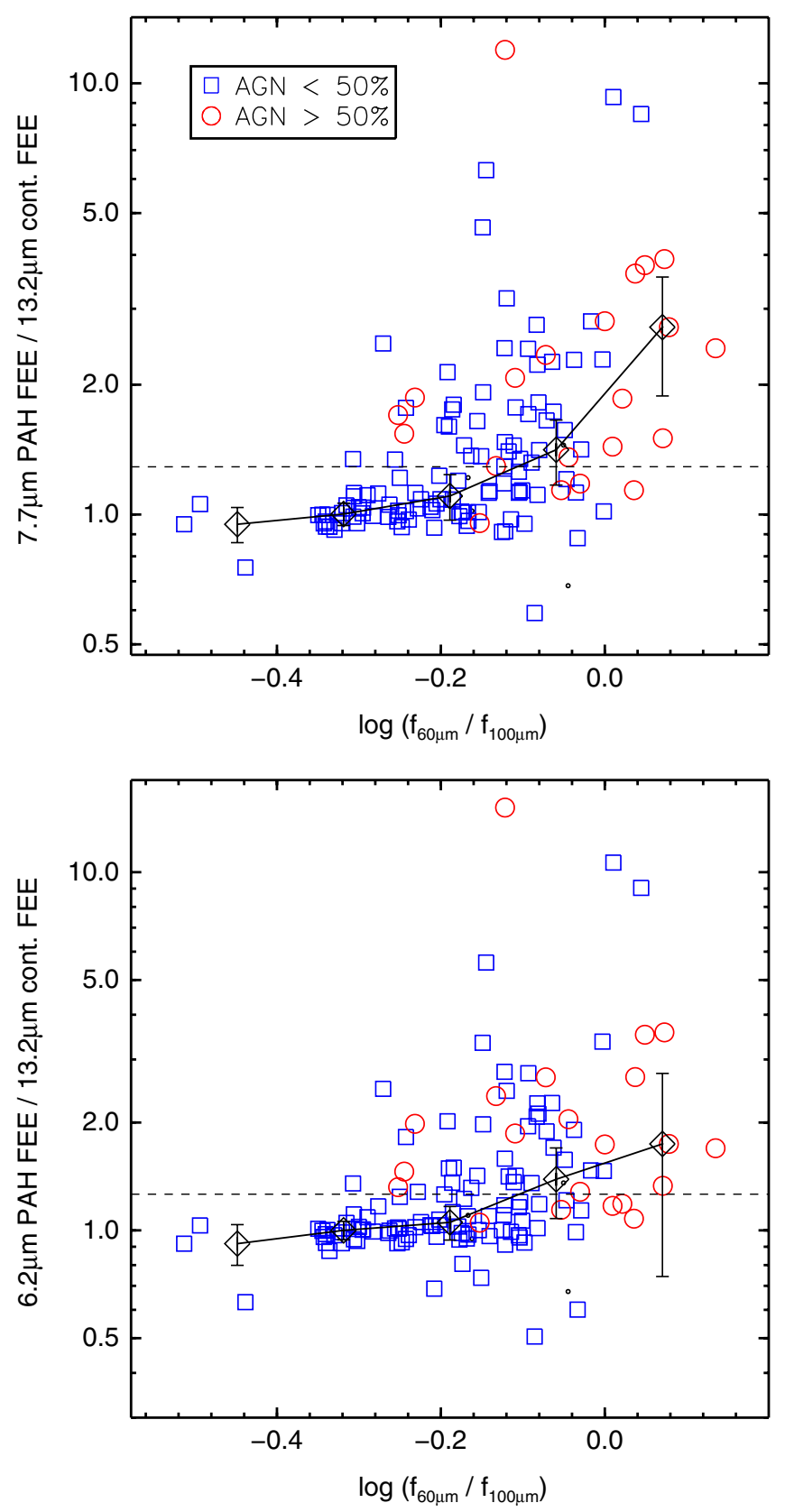

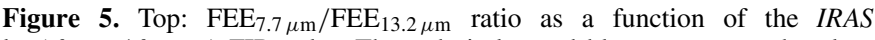
$\log \left(f_{60 \mu \mathrm{m}} / f_{100 \mu \mathrm{m}}\right)$ FIR color. The red circles and blue squares mark galaxies whose MIR AGN fraction is $\geqslant 0.5$ and $<0.5$, respectively. The small black dots represent sources for which the MIR AGN fraction is not available. The dashed line is defined as the median PAH-to-continuum FEE ratio value $+2 \sigma$ of star-formation-dominated sources (see Figure 4). Bottom: same plot but for the $\mathrm{FEE}_{6.2} \mu \mathrm{m} / \mathrm{FEE}_{13.2} \mu \mathrm{m}$ ratio.

(A color version of this figure is available in the online journal.)

PAH emission or that the PAHs are more extended in absolute terms in these galaxies. It is the MIR continuum that is more compact due to the enhancement caused by the nuclear power source that increases the PAH-to-continuum FEE ratio. It is important to stress that these compact galaxy cores are not only observed in ULIRGs (see, e.g., Charmandaris et al. 2002), we also see them in LIRGs (see also Figure 3). In addition, Figures 4 and 5 also suggest that irrespective of whether the nuclear MIR emission of (U)LIRGs is dominated by an AGN or a nuclear starburst, their impact on the compactness of the MIR continuum and PAH emission is the same. In other words, both processes 


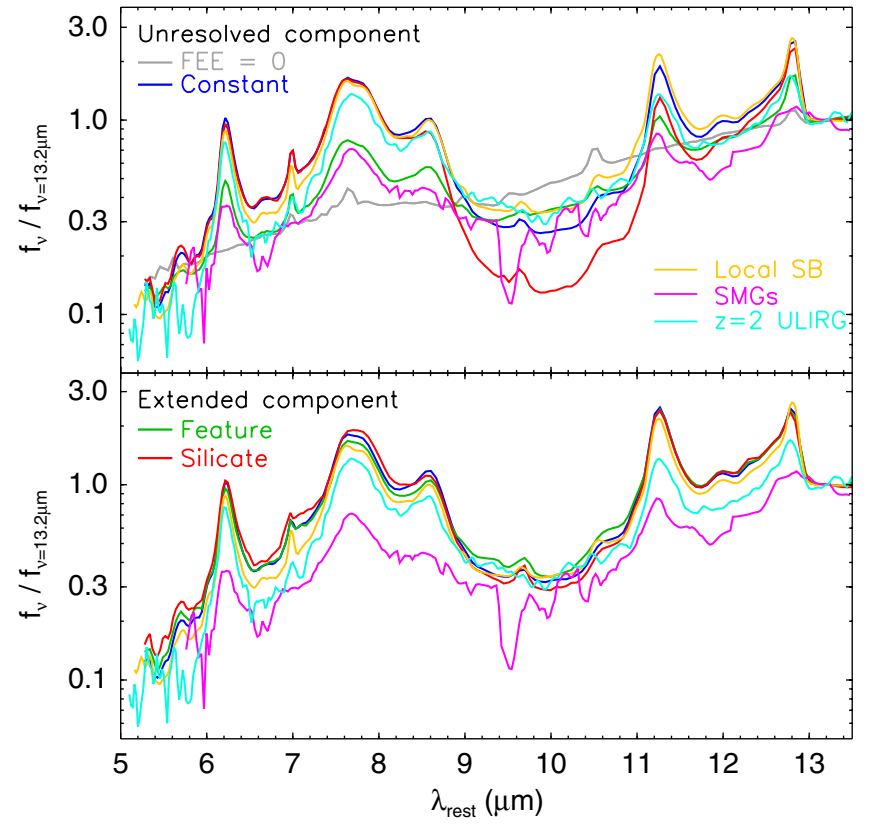

Figure 6. Weighted averaged spectra of the different $\mathrm{FEE}_{\lambda}$ types scaled to $13.2 \mu \mathrm{m}$ (unresolved: gray; constant/featureless: blue; PAH-dominated: green; silicate-dominated: red). The nuclear and extended emission components of each type are presented in the top and bottom panels, respectively. On both panels we overplot the local spectral template of Brandl et al. (2006) of normal star-forming galaxies in yellow, the averaged spectrum of the high-redshift submillimeter galaxies from Menéndez-Delmestre et al. (2009) in pink, as well as the template of the $z \sim 2$ ULIRGs from Farrah et al. (2008) in cyan. Unlike our GOALS galaxies, for which we show their nuclear and extended components, the comparison spectra are from the integrated emission of galaxies.

(A color version of this figure is available in the online journal.)

are indistinguishable in terms of the relative spatial extent of the PAH and MIR continuum emission.

We note that the MIR emission of $27 \%$ of the warm compact sources, those with $\log \left(f_{60 \mu \mathrm{m}} / f_{100 \mu \mathrm{m}}\right)>-0.2$ and above the dashed lines in the figures, is dominated by an AGN (MIR AGN fraction $\geqslant 0.5)$. It is known that AGN and star formation dominates the emission mainly at different wavelengths, i.e., the FIR colors of these galaxies are not affected by the existence of an AGN but they are associated principally to their star formation processes. This implies that in these (U)LIRGs, nuclear compact star formation, traced by their warm FIR colors, must be coexisting with MIR-detected AGN at the same time (see, e.g., Schweitzer et al. 2006; Netzer et al. 2007; Veilleux et al. 2009), both contributing to the large PAH-to-continuum FEE ratios they show.

\subsection{Averaged Spectra of Unresolved and Extended Components}

The technique we use to calculate the $\mathrm{FEE}_{\lambda}$ from the twodimensional Spitzer/IRS images allows us to derive not only the spatial extent of specific spectral features but also to separate the MIR spectrum of the extended emission component of galaxies from that of the unresolved component in a simple manner. This can be done just by multiplying their total, flux-calibrated Spitzer/IRS spectrum calculated by Petric et al. (2011), by their $\mathrm{FEE}_{\lambda}$ and $1-\mathrm{FEE}_{\lambda}$ functions, respectively. More technical details are available in the Appendix of Paper I.

Using this method, we display together in Figure 6 the averaged spectra of the different $\mathrm{FEE}_{\lambda}$ types for the unresolved and extended components of (U)LIRGs. The component averages were weighted by the mean $\mathrm{FEE}_{\lambda}$ and $1-\mathrm{FEE}_{\lambda}$ of each galaxy, respectively. This was done in order to give proportionally more importance in the calculation of the extended component averages to (U)LIRGs showing larger values of FEE and, on the other hand, more importance to (U)LIRGs showing lower values of FEE when the unresolved component averages are calculated. In any case, we note that the results are the same irrespectively of this weighting.

Strikingly, we find that the intensity of the spectral features (PAHs, emission lines, and $9.7 \mu \mathrm{m}$ silicate absorption) of the extended components are very similar in all $\mathrm{FEE}_{\lambda}$ types, which suggests that the global physical properties of the star formation in the external parts (disks) of galaxies $(d \gtrsim 1.5 \mathrm{kpc})$ are likely the same. Moreover, the averaged spectra of the extended emission of all $\mathrm{FEE}_{\lambda}$ types resemble that of the template of lower IR luminosity starburst galaxies from Brandl et al. (2006). On the other hand, the spectra of the unresolved component are substantially different among the three $\mathrm{FEE}_{\lambda}$ types even though the $f_{13.2 \mu \mathrm{m}} / f_{5.5 \mu \mathrm{m}}$ continuum ratio is similar in all of them, ranging between $\sim 5$ and 6 . The nuclear MIR spectra of the GOALS galaxy sample will be analyzed in detail in a forthcoming work of S. Stierwalt et al. (2011, in preparation). From another point of view, these results also imply that, on average, the differences seen in the global/integrated MIR spectra of local ULIRGs and also LIRGs arise only from the processes that are taking place in their cores, while the extended star formation appear not to be affected by the nuclear activity (see also Kaviraj 2009) and is similar to the more quiescent mode of star formation found in lower IR luminosity systems.

The difference between the MIR spectra of the unresolved emission of the constant and silicate-dominated $\mathrm{FEE}_{\lambda}$ types can be explained in terms of dust obscuration, with the silicatedominated $\mathrm{FEE}_{\lambda}$ type having a larger $9.7 \mu \mathrm{m}$ optical depth. The relative emission of the PAHs with respect to that of the continuum in the unresolved spectrum of the PAH-dominated $\mathrm{FEE}_{\lambda}$ type is lower than in the constant/featureless $\mathrm{FEE}_{\lambda}$ type. This implies that a mechanism, such as an AGN or a compact nuclear starburst, is either destroying the PAH carriers in the nuclei of these galaxies, or probably simply diluting their strength (peak to continuum ratio) by heating the small dust grains to higher temperatures thus increasing the MIR continuum emission; a result that is in agreement with Sections 3.3 and 3.4.

In Figure 7 we show the averaged spectra of the total (blue), unresolved nuclear (red), and extended (green) emission for the galaxies in our sample corresponding to each $\mathrm{FEE}_{\lambda}$ type. In Table 2 we present the silicate strength $\left(S_{\mathrm{Si} 9.7 \mu \mathrm{m}}\right)$ and $6.2 \mu \mathrm{m}$ PAH EW of the different averaged spectra. The $S_{\mathrm{Si} 9.7 \mu \mathrm{m}}$ was calculated by fitting a power law to the spectra, with anchors at $5.5 \mu \mathrm{m}, 6.6 \mu \mathrm{m}$, and $13.2 \mu \mathrm{m}$, and evaluated at its maximum. The $6.2 \mu \mathrm{m}$ PAH EW was obtained by fitting the local continuum of the feature using a linear function with anchors at $5.8 \mu \mathrm{m}$ and $6.6 \mu \mathrm{m}$ and integrating its emission from 5.9 to $6.5 \mu \mathrm{m}$.

By definition, the averaged spectra of the total as well as the unresolved and extended components for the constant/ featureless $\mathrm{FEE}_{\lambda}$ type galaxies are very similar. The silicate strengths vary between -0.8 and -1.00 (see Table 2), and the $6.2 \mu \mathrm{m}$ PAH EWs $\sim 0.5 \mu \mathrm{m}$, which are close to the values found for the starburst galaxy sample of Brandl et al. (2006). The average spectrum of the extended emission component for the PAH-dominated $\mathrm{FEE}_{\lambda}$ type, on the other hand, is very different from those of the unresolved and total components. Although the $S_{\mathrm{Si} 9.7 \mu \mathrm{m}}$ is similar, the $6.2 \mu \mathrm{m}$ PAH EW of 
Table 2

Silicate Strengths and PAH EWs of the $\mathrm{FEE}_{\lambda}$ Types

\begin{tabular}{|c|c|c|c|c|c|c|}
\hline \multirow{2}{*}{$\begin{array}{l}\mathrm{FEE}_{\lambda} \\
\text { Type } \\
(1)\end{array}$} & \multicolumn{2}{|c|}{ Total } & \multicolumn{2}{|c|}{ Unresolved } & \multicolumn{2}{|c|}{ Extended } \\
\hline & $\begin{array}{l}S_{\mathrm{Si} 9.7 \mu \mathrm{m}} \\
\quad(2)\end{array}$ & $\begin{array}{c}6.2 \mu \mathrm{m} \text { PAH EW } \\
\text { (3) }\end{array}$ & $\begin{array}{l}S_{\mathrm{Si} 9.7 \mu \mathrm{m}} \\
\quad(2)\end{array}$ & $\begin{array}{c}6.2 \mu \mathrm{m} \text { PAH EW } \\
\text { (3) }\end{array}$ & $\begin{array}{l}S_{\mathrm{Si} 9.7 \mu \mathrm{m}} \\
\quad \text { (2) }\end{array}$ & $\begin{array}{c}6.2 \mu \mathrm{m} \text { PAH EW } \\
\text { (3) }\end{array}$ \\
\hline Unresolved & $-0.55 \pm 0.01$ & $0.05 \pm 0.01$ & $-0.31 \pm 0.01$ & $0.00 \pm 0.01$ & $\ldots$ & $\ldots$ \\
\hline Constant/featureless & $-0.89 \pm 0.01$ & $0.50 \pm 0.01$ & $-1.01 \pm 0.01$ & $0.50 \pm 0.02$ & $-0.81 \pm 0.02$ & $0.54 \pm 0.01$ \\
\hline PAH-dominated & $-0.74 \pm 0.01$ & $0.29 \pm 0.01$ & $-0.60 \pm 0.03$ & $0.24 \pm 0.01$ & $-0.77 \pm 0.01$ & $0.48 \pm 0.01$ \\
\hline Silicate-dominated & $-1.53 \pm 0.02$ & $0.34 \pm 0.02$ & $-1.72 \pm 0.02$ & $0.43 \pm 0.02$ & $-0.94 \pm 0.01$ & $0.51 \pm 0.01$ \\
\hline
\end{tabular}

Notes. (1) $\mathrm{FEE}_{\lambda}$ type; (2) silicate strength; (3) $6.2 \mu \mathrm{m}$ PAH EW.

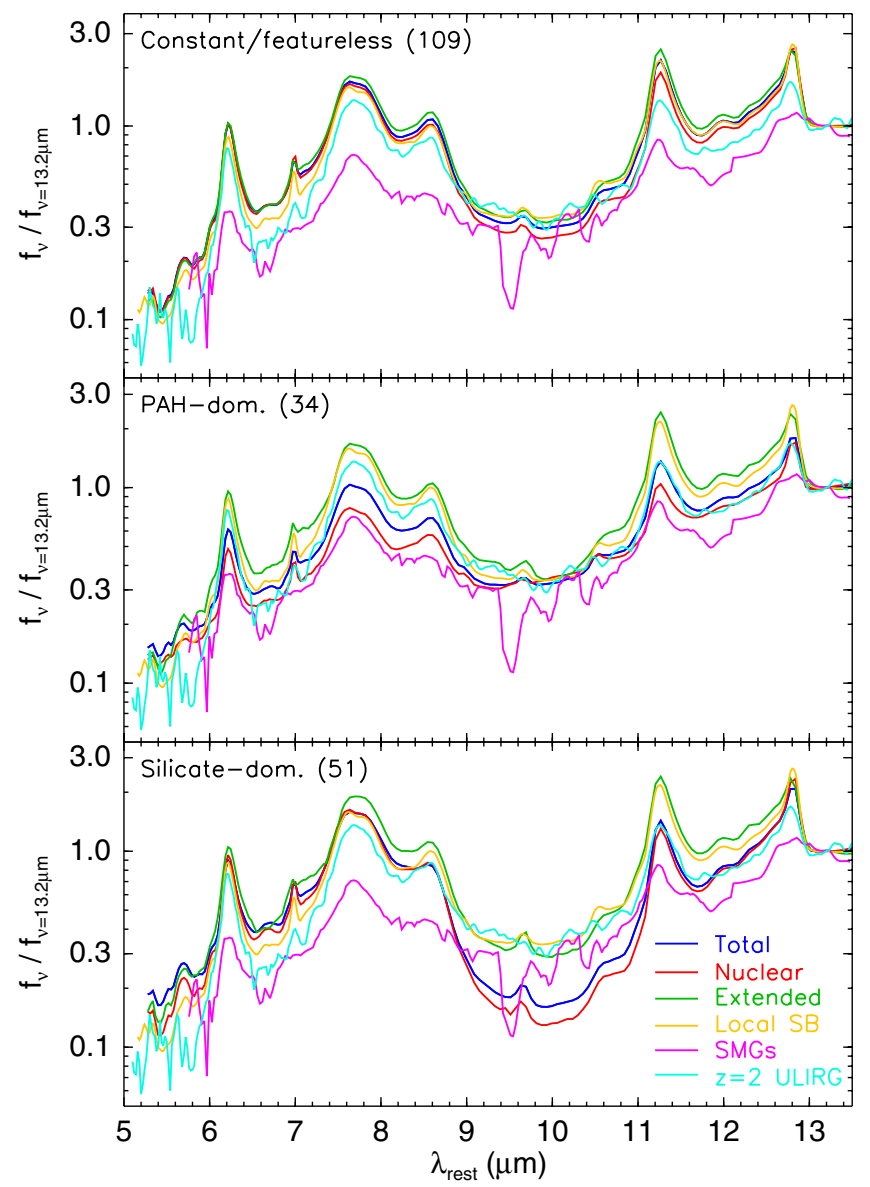

Figure 7. Averaged spectra of the total (blue), unresolved (red), and extended (green) emission components of our sample, normalized at $13.2 \mu \mathrm{m}$, for the different types of $\mathrm{FEE}_{\lambda}$. The integrated spectral templates used to compare our sample with low- and high-redshift star-forming galaxies are as in Figure 6. A total of 194 sources, for which both their $\mathrm{FEE}_{13.2 \mu \mathrm{m}}$ and $1-\mathrm{FEE}_{13.2 \mu \mathrm{m}}$ are larger than 0.05 and therefore could be normalized at $13.2 \mu \mathrm{m}$, were used for this plot.

(A color version of this figure is available in the online journal.)

the extended spectrum $(0.48 \mu \mathrm{m})$ is around twice that of the unresolved and total spectra $(0.24-0.29 \mu \mathrm{m}$; see Table 2$)$. The average $f_{13.2 \mu \mathrm{m}} / f_{5.5 \mu \mathrm{m}}$ continuum ratios of the nuclear and extended spectra are also very similar $\sim 7$. The averaged spectra of the components of the silicate-dominated $\mathrm{FEE}_{\lambda}$ type are intrinsically different. The spectrum of the unresolved emission shows a deep silicate absorption feature $\left(S_{\mathrm{Si} 9.7 \mu \mathrm{m}}=-1.72\right)$ while the spectrum of the extended emission is significantly shallower $\left(S_{\mathrm{Si} 9.7 \mu \mathrm{m}}=-0.94\right)$ but similar to the values found for the extended components of the constant/featureless and $\mathrm{PAH}$-dominated $\mathrm{FEE}_{\lambda}$ types. If we define $\Delta S_{\mathrm{Si}} 9.7 \mu \mathrm{m}$ as the difference between the silicate strength of the nuclear and extended component of a galaxy, then $\Delta S_{\mathrm{Si} 9.7 \mu \mathrm{m}}=-0.78$ for the silicate-dominated $\mathrm{FEE}_{\lambda}$ type (see also Section 3.2). The averaged nuclear spectra of the unresolved $\mathrm{FEE}_{\lambda}$ type (Figure 6) show an almost flat rising continuum, a moderate silicate strength $\left(S_{\mathrm{Si} 9.7 \mu \mathrm{m}}=-0.31\right)$, and almost no PAH emission $(6.2 \mu \mathrm{m}$ PAH EW $\sim 0.00 \mu \mathrm{m})$, all of them typical signatures of AGN-dominated galaxies. Their MIR spectrum is similar to sources in region 1A of the Spoon et al. (2007) diagram, in which the AGN emission is the likely culprit for the dilution of the PAH features.

The classification of the GOALS galaxies into different stages of interaction carried out by Petric et al. (2011) and Haan et al. (2011) also allows us to explore whether the PAH-to-continuum FEE ratios or the MIR spectral shape of the nuclear and extended components of galaxies are related to the merger stage of the systems. Unfortunately, no such clear trend is found. Only at the final stage of interaction (stage 4), when the nuclei of the galaxies are already assembled, the averaged spectrum of their unresolved component shows a deeper silicate absorption feature than that displayed by the spectrum of the extended component. This is understood if we take into account that most of the ULIRGs in GOALS, which show the largest silicate strengths, are classified as being in this latest merger stage. On the other hand, the averaged spectra of both components for galaxies grouped in stages $0-3$ are very similar, and resemble that of local starburst galaxies.

\subsection{High-redshift Implications}

In Figures 6 and 7 we also compare the averaged spectra of the GOALS sample, both nuclear and extended components, with the composite, integrated spectra of the $z \sim 2$ ULIRG sample from Farrah et al. (2008), and the submillimeter galaxies (SMGs) studied in Menéndez-Delmestre et al. (2009) which are located at a redshift range of $z \sim 0.65-3.2$. As already mentioned in Farrah et al. (2008), their composite spectrum of high- $z$ ULIRGs and HyperLIRGs $\left(L_{\mathrm{IR}}=10^{12.9}-10^{13.8} L_{\odot}\right)$ is very similar to that of the starburst galaxies from Brandl et al. (2006). We also note that it is also very similar to the averaged spectra of the extended components of our galaxy sample. This suggests that the properties of star-forming high- $z$ ULIRGs, often selected based on the characteristic Spitzer/Infrared Array Camera (IRAC) colors due to the $1.6 \mu \mathrm{m}$ stellar bump and their Spitzer/MIPS $24 \mu \mathrm{m}$ fluxes (e.g., Magdis et al. 2010), are very similar to the extended disks of local (U)LIRGs. However, the composite, integrated SMG spectrum is different from our average spectrum of the extended component, displaying PAHs with a considerably lower EW. Menéndez-Delmestre et al. (2009) argued that an additional power-law-type source of hot dust emission was needed to partially enhance the MIR 
continuum of these galaxies. They suggested that this would arise either from an AGN torus or from optically thick dust emission around star-forming regions, in agreement with the scenario we propose for the compact nuclear sources in the previous sections. Interestingly, the SMG composite spectrum is more similar to the averaged nuclear spectrum of (U)LIRGs belonging to the $\mathrm{PAH}$-dominated $\mathrm{FEE}_{\lambda}$ galaxy type (see $\mathrm{S}$. Stierwalt et al. 2011, in preparation). We also note that while the $[\mathrm{Ne}$ II] $12.81 \mu \mathrm{m}$ emission line is clearly visible in all of our averaged spectra as well as in the high- $z$ ULIRG spectrum, it is almost absent from that of the SMGs. Nevertheless, we attribute this difference to the limited quality of the composite spectrum of the SMGs, which prevent us from performing meaningful comparisons regarding specific features in the spectra.

Summarizing, the spectra of the extended components of (U)LIRGs are very similar independently of their $\mathrm{FEE}_{\lambda}$ type, suggesting that the extended disk emission of all LIRGs and ULIRGs could be dominated by PAHs excited principally by non-ionizing stars and quiescent star-forming regions. The fact that recent Herschel observations imply that the galactic disks are responsible for the bulk of the diffuse cold dust emission peaking at 100-500 $\mu \mathrm{m}$ (Bendo et al. 2010) leads us to speculate whether this would also hold in (U)LIRGs. Within this picture, their outer disks, traced by the PAH spatial profiles, would be responsible for the cold FIR emission. Meanwhile, the inner nuclei of the most compact sources, where most of their energy is produced, would dominate the MIR continuum and also contribute to show warmer FIR colors than galaxies without compact cores. Due to their distance and small physical size this cannot be verified directly by Herschel or even future missions, such as the Space Infrared Telescope for Cosmology and Astrophysics. However, modeling the FIR line emission (e.g., van der Werf et al. 2010; González-Alfonso et al. 2010) will enable us to probe the excitation and density of gas and thus provide further constrains on its spatial distribution.

\section{CONCLUSIONS}

We analyzed the spatial profiles of low spectral resolution 5-14 $\mu \mathrm{m}$ Spitzer/IRS spectra of the GOALS galaxy sample and quantified the spatial extent of their MIR emission, $\mathrm{FEE}_{\lambda}$. Our work indicates the following.

1. The $[\mathrm{Ne}$ II] $12.81 \mu \mathrm{m}$ emission is as compact as the MIR continuum, but the PAHs are more extended in many galaxies. This is in agreement with studies showing that the [Ne II] $12.81 \mu \mathrm{m}$ and MIR continuum emissions trace the ionizing stars (i.e., clumpy, current star formation), while the PAH emission is more representative of older, colder, and diffuse stellar populations.

2. The $11.3 \mu \mathrm{m}$ PAH emission is more extended than that of the $6.2 \mu \mathrm{m}$ and $7.7 \mu \mathrm{m}$ PAHs, which is consistent with the formers being enhanced in a more ionized medium while the $11.3 \mu \mathrm{m}$ PAH emission is similar for neutral and ionized molecules.

3. On average, the MIR continuum becomes more compact than the PAH emission as the MIR is increasingly dominated by an AGN. That is, the presence of an AGN modifies the spatial extent of the hot dust continuum but has a negligible effect on the overall, kpc-scale distribution of the PAH emission. None of the AGN-dominated (U)LIRGs shows a constant/featureless $\mathrm{FEE}_{\lambda}$ type, implying that the AGN leaves a "footprint" in the $\mathrm{FEE}_{\lambda}$ function of a galaxy. Therefore, the MIR emission of the galaxies showing a con- stant $\mathrm{FEE}_{\lambda}$ will not be dominated by an AGN or, in general, show compact MIR continuum emission.

4. The PAH-to-continuum FEE ratio of (U)LIRGs increases as the global dust temperature, traced by their IRAS $\log \left(f_{60 \mu \mathrm{m}} / f_{100 \mu \mathrm{m}}\right)$ FIR color, becomes warmer. This can be attributed to the presence of a compact, powerful (circum-) nuclear starburst in these galaxies which would increase the temperature of the dust, and also the ratio between the volume of the shells of the PDRs-from where PAH emission arises - and the volume of the hot dust-emitting region. "Normal" galaxies do not show enhanced PAH-tocontinuum FEE ratios but a rather constant value. On the other hand, in (U)LIRGs with large PAH-to-continuum FEE ratios the MIR continuum is more compact than the PAH emission, which is still as extended as in the "normal" population since it arises mostly from a diffuse component (their disks). Nonetheless, in terms of the relative spatial distribution of the PAH and continuum emissions, this effect is indistinguishable from that caused by an AGN. Moreover, both processes, compact star formation and AGN activity, coexist in $27 \%$ of our (U)LIRGs sample.

5. The intensity of the spectral features and continuum emission of the extended emission component of all (U)LIRGs are very similar, indicating that the properties of the star formation in the external parts of galaxies (disks; $d \gtrsim 1.5 \mathrm{kpc}$ ) are uniform. Moreover, they are similar to local starburst galaxies as well as to $z \sim 2$ ULIRGs (selected by their Spitzer/IRAC and MIPS colors), but different from submillimeter-selected high-redshift galaxies. On the other hand, the MIR spectra of the unresolved nuclear component of galaxies vary widely.

6. These results imply that, on average, the diversity seen in the properties of global/integrated MIR spectra of local ULIRGs as well as LIRGs arise only from the processes that are taking place in their cores. The extended star formation appears unaffected by the nuclear activity and it is similar to the more quiescent mode of star formation found in lower IR luminosity systems. We speculate that the outer disks/ regions of (U)LIRGs, traced by their PAH spatial profiles, are responsible for most of the cold FIR emission as seen in galaxies with lower IR luminosities, while the nuclei of the most compact (U)LIRGs, as stated in Díaz-Santos et al. (2010b), would dominate the MIR continuum and contribute to show overall warmer FIR colors.

We thank the referee for her/his useful comments which helped to improved the paper. T.D.-S. thanks D. Elbaz, E. Le Floc'h, V. Lebouteiller, E. Daddi, and G. Magdis for stimulating discussions, as well as all colleagues at CEA/Saclay (France), where part of this work was done, for their hospitality. T.D.-S. and V.C. acknowledge partial support from the EU ToK grant 39965 and FP7-REGPOT 206469. This research has made use of the NASA/IPAC Extragalactic Database (NED), which is operated by the Jet Propulsion Laboratory, California Institute of Technology, under contract with the National Aeronautics and Space Administration, and of NASA's Astrophysics Data System (ADS) abstract service.

\section{REFERENCES}

Allamandola, L. J., Hudgins, D. M., \& Sandford, S. A. 1999, ApJ, 511, L115 Alonso-Herrero, A., Rieke, G. H., Rieke, M. J., \& Scoville, N. Z. 2002, AJ, 124, 166

Armus, L., Charmandaris, V., Bernard-Salas, J., et al. 2007, ApJ, 656, 148 
Armus, L., Mazzarella, J. M., Evans, A. S., et al. 2009, PASP, 121, 559 Bendo, G. J., Wilson, C. D., Pohlen, M., et al. 2010, A\&A, 518, L65 Brandl, B. R., Bernard-Salas, J., Spoon, H. W. W., et al. 2006, ApJ, 653, 1129

Calzetti, D., Kennicutt, R. C., Engelbracht, C. W., et al. 2007, ApJ, 666, 870

Caputi, K. I., Lagache, G., Yan, L., et al. 2007, ApJ, 660, 97

Charmandaris, V., Laurent, O., Le Floc'h, E., et al. 2002, A\&A, 391, 429

Charmandaris, V., Le Floc'h, E., \& Mirabel, I. F. 2004, ApJ, 600, L15

Díaz-Santos, T., Alonso-Herrero, A., Colina, L., et al. 2008, ApJ, 685, 211

Díaz-Santos, T., Alonso-Herrero, A., Colina, L., et al. 2010a, ApJ, 711, 328

Díaz-Santos, T., Charmandaris, V., Armus, L., et al. 2010b, ApJ, 723, 993

Draine, B. T., \& Li, A. 2001, ApJ, 551, 807

Farrah, D., Lonsdale, C. J., Weedman, D. W., et al. 2008, ApJ, 677, 957

Galliano, F., Madden, S. C., Tielens, A. G. G. M., Peeters, E., \& Jones, A. P. 2008, ApJ, 679, 310

García-Marín, M., Colina, L., \& Arribas, S. 2009, A\&A, 505, 1017

Genzel, R., Lutz, D., Sturm, E., et al. 1998, ApJ, 498, 579

González-Alfonso, E., Fischer, J., Isaak, K., et al. 2010, A\&A, 518, L43

Haan, S., Surace, J. A., Armus, L., et al. 2011, AJ, 141, 100

Howell, J. H., Armus, L., Mazzarella, J. M., et al. 2010, ApJ, 715, 572

Imanishi, M. 2009, ApJ, 694, 751

Imanishi, M., Dudley, C. C., Maiolino, R., et al. 2007, ApJS, 171, 72

Imanishi, M., Maiolino, R., \& Nakagawa, T. 2010, ApJ, 709, 801

Kaviraj, S. 2009, MNRAS, 394, 1167

Kramer, C., Buchbender, C., Xilouris, E. M., et al. 2010, A\&A, 518, L67

Laurent, O., Mirabel, I. F., Charmandaris, V., et al. 2000, A\&A, 359, 887

Le Floc'h, E., Papovich, C., Dole, H., et al. 2005, ApJ, 632, 169

Lutz, D., Spoon, H. W. W., Rigopoulou, D., Moorwood, A. F. M., \& Genzel, R. 1998b, ApJ, 505, L103

Magdis, G. E., Elbaz, D., Hwang, H. S., et al. 2010, MNRAS, 409, 22
Magnelli, B., Elbaz, D., Chary, R. R., et al. 2011, A\&A, 528, A35

Menéndez-Delmestre, K., Blain, A. W., Smail, I., et al. 2009, ApJ, 699, 667

Murphy, E. J., Chary, R., Dickinson, M., et al. 2011, ApJ, 732, 126

Netzer, H., Lutz, D., Schweitzer, M., et al. 2007, ApJ, 666, 806

Pereira-Santaella, M., Alonso-Herrero, A., Rieke, G. H., et al. 2010, ApJS, 188 447

Pérez-González, P. G., Rieke, G. H., Egami, E., et al. 2005, ApJ, 630, 82

Petric, A. O., Armus, L., Howell, J., et al. 2011, ApJ, 730, 28

Rujopakarn, W., Rieke, G. H., Eisenstein, D. J., \& Juneau, S. 2011, ApJ, 726, 93

Sales, D. A., Pastoriza, M. G., \& Riffel, R. 2010, ApJ, 725, 605

Sanders, D. B., Mazzarella, J. M., Kim, D.-C., Surace, J. A., \& Soifer, B. T. 2003, AJ, 126, 1607

Sanders, D. B., \& Mirabel, I. F. 1996, ARA\&A, 34, 749

Sauvage, M., Sacchi, N., Bendo, G. J., et al. 2010, A\&A, 518, L64

Sauvage, M., \& Thuan, T. X. 1992, ApJ, 396, L69

Schweitzer, M., Lutz, D., Sturm, E., et al. 2006, ApJ, 649, 79

Smith, J. D. T., Dale, D. A., Armus, L., et al. 2004, ApJS, 154, 199

Smith, J. D. T., Draine, B. T., Dale, D. A., et al. 2007, ApJ, 656, 770

Soifer, B. T., Bock, J. J., Marsh, K., et al. 2003, AJ, 126, 143

Soifer, B. T., Neugebauer, G., Matthews, K., Egami, E., \& Weinberger, A. J. 2002, AJ, 124, 2980

Spoon, H. W. W., Marshall, J. A., Houck, J. R., et al. 2007, ApJ, 654, L49

van der Werf, P. P., Isaak, K. G., Meijerink, R., et al. 2010, A\&A, 518, L42

Veilleux, S., Kim, D., \& Sanders, D. B. 2002, ApJS, 143, 315

Veilleux, S., Rupke, D. S. N., Kim, D.-C., et al. 2009, ApJS, 182, 628

Walterbos, R. A. M., \& Greenawalt, B. 1996, ApJ, 460, 696

Wu, Y., Charmandaris, V., Huang, J., Spinoglio, L., \& Tommasin, S. 2009, ApJ, 701,658 